\title{
A mathematical model of human inhibitory control
}

\author{
Kyle Lyman ${ }^{1 *}$, Joaquin Anguera², Adam Gazzaley², David Terman ${ }^{1}$ \\ From Nineteenth Annual Computational Neuroscience Meeting: CNS*2010 \\ San Antonio, TX, USA. 24-30 July 2010
}

The Stop-Signal paradigm has generated a great deal of research interest among cognitive neuroscientists because of its ability to probe response inhibition [1]. In the task, subjects make a speeded button press when prompted, except on a fraction of trials where a second stimulus (the stop signal) instructs the subject to withold the prepotent response. The success or failure of the subject on trial ' $n$ ' is known to influence the following ' $n+1$ ' trial, and is thought to index top-down control [1-3]. In the present study, we examined the putative networks involved in action monitoring [4] and top down control [5] with the goal of understanding the neural mechanisms underlying this stop signal aftereffect [6]. We utilize a dynamical systems approach based on a recent conductance-based model of prefrontal cortex activity [7]. The behavior of the model is dictated by the amount of inhibitory control utilized during trial ' $n$ ', with successful inhibition requiring the most inhibitory resources and go trials (no stop signal) the least, modeled here as recurrent activity within the prefrontal cortex. The use of a biophysically realistic model facilitated testing the hypothesis that a prefrontal topdown signal drives the extent of response inhibition on subsequent trials.

A single trial in the model begins with the presentation of a 'GO' stimulus to a 'GO' neural group. During a non-stop signal trial, activity within the GO group increases until it reaches a fixed threshold and we define the model to have 'gone'. During a stop signal trial, an 'Inhibition' neural group (as in [5]) begins to generate activity. Depending on the stop signal delay, the model either goes (failed inhibition) or successfully inhibits. Activation of these inhibitory groups also activates another neural group that represents how the anterior

\footnotetext{
* Correspondence: KyleLyman@gmail.com

'Department of Mathematics, Ohio State University, Columbus, $\mathrm{OH} 43201$, USA
}

cingulate cortex (ACC, as in [4]) monitors conflict with respect to the different time courses of the 'Inhibition' and 'GO' groups. As suggested in [3-5], ACC activity is passed onto the prefrontal cortex (modeled per [7]) where recurrent activity is maintained to influence the following trials. The model exhibits realistic patterns of reaction times, with successful inhibition trials eliciting the most post-trial slowing, then failed inhibition trials, then no stop signal trials. These findings suggest that inhibitory after-effects may be driven by ACC and prefrontal influences, with the proposed mechanism potentially having implications for situations where motor inhibition is impaired, such as schizophrenia and aging.

\section{Author details}

${ }^{1}$ Department of Mathematics, Ohio State University, Columbus, $\mathrm{OH} 43201$, USA. ${ }^{2}$ Department of Neurology and Physiology, University of California San Francisco, San Francisco, California, USA.

Published: 20 July 2010

\section{References}

1. Logan GD, Cowan WB: On the ability to inhibit thought and action - A theory of an act of control. Psychological Review 1984, 91:295-327.

2. Rieger M, Gauggel S: Inhibitory after-effects in the stop signal paradigm. British Journal of Psychology 1999, 90:509-518.

3. Aron A: The neural basis of inhibition in cognitive control. Neuroscientist 2007, 13(3):214-228.

4. Botvinick MM, Braver TS, Carter CS, Barch DM, Cohen JD: Conflict monitoring and cognitive control. Psychological Review 2001, 108(3):624-652.

5. Lo CC, Boucher L, Paré M, Schall JD, Wang X-J: Proactive inhibitory control and attractor dynamics in countermanding action: a spiking neural circuit model. J. Neurosci 2009, 29:9059-9071.

6. Anguera JA, Lyman K, Gazzaley A: Neural correlates of response inhibition after-effects. Published Human Brain Mapping Conference 2010, Barcelona Spain.

7. Lyman K, McDougal R, Myers B, Tien J, Zeki M, Fall C, Terman D: A working memory model based on excitatory-inhibitory interactions and calcium dynamics., Published abstract, Presented at this conference.

doi:10.1186/1471-2202-11-S1-P82

Cite this article as: Lyman et al:: A mathematical model of human inhibitory control. BMC Neuroscience 2010 11(Suppl 1):P82. 